\title{
Revisited Kinetic Chain Length and Reactivity Ratio Approaches for Linear Polymers and Copolymers
}

\author{
Mohammad M. Fares*
}

Dept. of Applied Chemistry, Jordan University of Science \& Technology, P.O. Box: 3030 22110, Irbid, Jordan

\begin{abstract}
Two revisited mathematical kinetic relationships were derived, evaluated and applied on linear polymers such as polystyrene and copolymers such as (NIPAAm-alt-HEMA) and (MAAm-alt-HEMA) copolymers respectively. The validity of these equations was successfully verified. The first relationship, equation 12, interrelates exponentially kinetic chain length, average molecular weight and degree of polymerization of linear polymers with different temperatures. Furthermore; equation 12 could novelly define the rate of polymerization $\left(\mathrm{R}_{\mathrm{p}}\right)$ and consequently the overall activation energy $(\Delta \mathrm{E})$ of the polymerization process could be determined. The second derived relationship interrelates the reactivity ratio product $\left(r_{1} r_{2}\right)$ of two monomers interacting with each other, with different temperature. Application of equation 16 could determine the behavioral sequence of monomer 1 toward monomer 2 in the copolymerization process. The value of $\left(E_{12}+E_{21}\right)-\left(E_{11}+E_{22}\right)$ could result with the determination of type of copolymers formed. The reactivity ratio values for (NIPAAm-alt-HEMA) and (MAAm-alt-HEMA) copolymers were determined using Kelen-Tudos technique.
\end{abstract}

Keywords; Kinetic chain length, reactivity ratio, Theoretical Kinetic approach.

\section{INTRODUCTION}

Radical kinetic chain polymerization, as one of the most crucial techniques in the control and optimization of recent polymeric industries, is always finding renewable generated ideas and interest in the theory and kinetic parameters of polymerization processes. This major concern is due to the outstanding applications, practical use, and relatively easy control [1] of radical reactions. This concern have further oriented us to derive practical, adequate and easily handled kinetic relationships for practical purposes. The determination of polymerization rate in equation 1 at different temperatures, in Arrhenius equation, implies the determination of $\mathrm{k}_{\mathrm{p}}, \mathrm{k}_{\mathrm{d}}$, and $\mathrm{k}_{\mathrm{t}}$ respectively.

$\ln \left(k_{p}\left(\frac{k_{d}}{k_{t}}\right)^{1 / 2}\right)=\ln \left(A_{p}\left(\frac{A_{d}}{A_{t}}\right)^{1 / 2}\right)-\frac{\left(E_{p}+\frac{E_{d}}{2}-\frac{E_{t}}{2}\right)}{R T}$

where $\mathrm{k}_{\mathrm{p}}, \mathrm{k}_{\mathrm{d}}$, and $\mathrm{k}_{\mathrm{t}}$ are rate constants for propagation, initiator dissociation and termination respectively. Since rate of polymerization $\left(\mathrm{R}_{\mathrm{p}}\right)$ is given by

$$
R_{p}=k_{p}\left(\frac{f k_{d}[I]}{k_{t}}\right)^{1 / 2}
$$

Substituting eq'n (2) in (1) yields;

$\ln R_{p}=\ln \left(A_{p}\left(\frac{A_{d}}{A_{t}}\right)^{1 / 2}\right)+\ln \left(f[I]^{1 / 2}[M]\right)-\frac{\Delta E_{p}}{R T}$

The exact determination of polymerization rate $\left(\mathrm{R}_{\mathrm{p}}\right)$ is the most important factor in Arrhenius equation, due to consequence determination of exact overall activation energy. In

*Address correspondence to this author at the Department of Applied Chemistry, Jordan University of Science \& Technology, P.O. Box: 3030 22110, Irbid, Jordan; E-mail: fares @just.edu.jo the literature $R_{p}$ were determined using spectroscopic means such as UV-Vis [2], FTIR [3], NMR [4], XRD [5], mass spectrometry [6] and TGA [9], or by Laser beam technology [8-9], or using conventional \%Conversion versus Time plot by precipitation of polymers [10], where $R_{p}$ is determined from the non-steady state curve.

Consequently the determination the overall activation energy $\boldsymbol{\Delta} \boldsymbol{E}_{a}=\boldsymbol{E}_{p}+\left(\boldsymbol{E}_{d} / \mathbf{2}\right)-\left(\boldsymbol{E}_{t} / \mathbf{2}\right)$ describes the kinetic changes in the polymerization process whether it shows an Arrhenius behavior (i.e. $\Delta \mathrm{E}_{\mathrm{a}}>0$ ), which implies that the reaction rate increase as a result of increasing temperature, or nonArrhenius behavior (i.e. $\Delta \mathrm{E}_{\mathrm{a}}<0$ ), which subsequently implies that reaction rate decreases as a result of increasing temperature. The overall activation energy is associated with $E_{p}$, $E_{d}$, and $E_{t}$ values, which dramatically change with many factors such as monomer structure and geometry, initiator type, temperature, polymerization time, and chain transfer agent.

The aim of this work is to derive, investigate and apply revisited kinetic relationships through raising a new definition of rate of polymerization $\left(\mathrm{R}_{\mathrm{p}}\right)$ for linear polymerization and copolymerization processes, applying that into Arrhenius pattern and eventually investigating the consistency and genuinity of such relationships.

\section{EXPERIMENTAL}

\section{Materials}

Styrene (99\%, Acros), Methacrylamide (MAAm) (99\%, Fluka), $N$-isopropylacrylamide (NIPAAm) $(99 \%$, Acros) and 2-Hydroxyethylmethacrylate (HEMA) (99\%, Acros) monomers were kept in refrigerator and used as received, $N, N, N^{\prime}, N^{\prime}$-Tetraethylenediamine (TEMED) (Sigma-Aldrich) used as an accelerator, was used as received, Potassium peroxodisulfate (KPS) (BDH chemicals Ltd), and benzoylperoxide as an initiator, was further purified by recrystallization. All solvents and other chemicals were of analytical grade. 


\section{Synthesis of Polystyrene (PS)}

Styrene monomer were conventionally polymerized under $\mathrm{N}_{2}$ atmosphere, in bulk, in the temperature range of 60$80{ }^{\circ} \mathrm{C}$ using $0.1 \%$ benzoylperoxide, where at each time interval 15, 30, 45, 60, 75 min., excess methanol nonsolvent were used to precipitate polystyrene. Continuous washing with methanol were done to remove monomer traces. Polystyrene were then dried and weighed. The weight average molecular weight was determined using light scattering technique [11]. The degree of polymerization were determined from the ratio of absorbance of aliphatic $\mathrm{v}_{\mathrm{C}=\mathrm{C}}$ of monomer before polymerization and after polymer precipitation.

\section{Synthesis of (NIPAAm-alt-HEMA) and (MAAm-alt- HEMA) Copolymers}

$0.66,1.0,1.3,1.5,3.0$, and 9.0 molar ratios of NIPAAm to HEMA (i.e. $\mathrm{M}_{\text {NIPAAm }} / \mathrm{M}_{\text {HEMA }}$ ) and MAAm to HEMA (i.e. $\left.\mathrm{M}_{\text {MAAm }} / \mathrm{M}_{\text {HEMA }}\right)$ as mentioned previously $[12,13]$, were synthesized as follow; each monomer concentration were dissolved in $25 \mathrm{ml}$ of de-ionized water. The solutions were sparged with $\mathrm{N}_{2}$ for 5 minutes or until the monomer is dissolved. To each solution a $5 \mathrm{ml}$ of $10 \%$ (wt/wt) KPS and 5 $\mathrm{ml}$ of $10 \%(\mathrm{wt} / \mathrm{wt})$ TEMED with respect to total monomers weights were added under $\mathrm{N}_{2}$ atmosphere. Then the mixture was set in $250 \mathrm{ml}$ round bottom flask and sealed under $\mathrm{N}_{2}$ atmosphere for 3.5 hours in water-bath fixed at $30^{\circ} \mathrm{C}$. After the copolymerization was complete, the product was poured to an excess of chloroform, stirred for $15 \mathrm{~min}$, and washed with hot de-ionized water to remove homopolymers, then filtered and dried in an oven at $80{ }^{\circ} \mathrm{C}$ for 8-12 hours. The samples were further purified via centrifugation. The weight average molecular weight was determined using light scattering technique [11]. Reactivity ratios $r_{1}$ and $r_{2}$ values were determined using Kelen-Tudos technique [14] at 20-40 ${ }^{\circ} \mathrm{C}$ temperature range for (NIPAAm-alt-HEMA) and at $5-20{ }^{\circ} \mathrm{C}$ temperature range for (MAAm-alt-HEMA) copolymers.

\section{THEORETICAL APPROACH (1)}

Kinetic chain length $\left(\boldsymbol{L}_{i, t}\right)$ is defined as the number of monomers added into a growing macroradical of $\boldsymbol{i}^{\text {th }}$ length in unit time interval. The molecular weight of this chain in the same time interval, $\boldsymbol{M}_{\boldsymbol{i}, \boldsymbol{t}}$, can be described as the kinetic chain length, $\boldsymbol{L}_{i, t}$, times the molar mass of the repeating unit , $\boldsymbol{M}_{\boldsymbol{r u}}$, as follows;

$$
M_{i, t}=L_{i, t} \times M_{r u}
$$

At time $\infty$, if theoretically assumed all monomers can join all different chains, the overall kinetic chain lengths is then called $\boldsymbol{L}_{\infty}$ and consequently the average molecular weight can also be called $\boldsymbol{M}_{\infty}$. Thus the fraction of reacted monomers joining the $\boldsymbol{i}^{\text {th }}$ length macroradical chain, $\boldsymbol{p}_{\boldsymbol{i}}$, can be presented by dividing the kinetic chain length of $i^{\text {th }}$ length macroradical chain, at certain time $\left(\boldsymbol{L}_{i, t}\right)$, to the overall kinetic chain lengths $\boldsymbol{L}_{\infty}$. Consequently this fraction can be descried by the ratio of molecular weight of $\boldsymbol{i}^{\text {th }}$ length macroradical chain at certain time $\left(\mathbf{M}_{\mathbf{i}, \mathbf{t}}\right)$ by the molecular weight $\boldsymbol{M}_{\infty}$. Thus the relation becomes;

$p_{i}=\frac{M_{i, t}}{M_{\infty}}=\frac{L_{i, t}}{L_{\infty}}$
The degree of polymerization $\left(\boldsymbol{D P} \boldsymbol{P}_{\boldsymbol{i}}\right)$ is defined as ratio of initial monomer conc., $[\boldsymbol{A}]_{0}$, to monomer conc. at polymerization time $\boldsymbol{t},[\boldsymbol{A}]_{t}$, (i.e. $\left.\boldsymbol{D} \boldsymbol{P}_{i}=[\boldsymbol{A}]_{0} /[\boldsymbol{A}]_{t}\right)$. The reciprocal of $D \boldsymbol{P}_{\boldsymbol{i}}$ presents the fraction of unreacted monomers (i.e. the monomers that did not join the $i^{\text {th }}$ length macroradical chain, where $\boldsymbol{i}$ present the number of repeating units in the macroradical chain). Hence, the fraction of reacted monomers join-

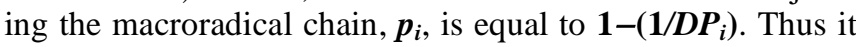
can be equalized with equation 5 as follows;

$$
\begin{aligned}
& \frac{M_{i, t}}{M_{\infty}}=1-\frac{1}{D P_{i}} \\
& M_{i, t}=M_{\infty} \cdot\left(1-\frac{1}{D P_{i}}\right)
\end{aligned}
$$

Number average molecular weight $\left(\overline{M_{n}}\right)$ [15] is defined in equation 7, where $\boldsymbol{M}_{\boldsymbol{i}}$ is the molecular weight of $\boldsymbol{i}^{\text {th }}$ length chain, $\boldsymbol{n}_{i}$ is number of chains with $\boldsymbol{i}^{\text {th }}$ length, and $\boldsymbol{N}_{T}$ is the total number of chains with different chain lengths. Thus the fraction $n_{i} / N_{T}$ represents the fraction of chains with $i^{\text {th }}$ length with respect to total different length chains.

$$
\begin{aligned}
& \overline{M_{n}}=\frac{\sum_{i} M_{i} \cdot n_{i}}{\sum_{i} n_{i}} \\
& =\frac{M_{1} n_{1}}{N_{T}}+\frac{M_{2} n_{2}}{N_{T}}+=\frac{M_{3} n_{3}}{N_{T}}+\ldots+\frac{M_{n} n_{n}}{N_{T}}
\end{aligned}
$$

Furthermore; it is known that different chain lengths (i.e. different molecular weight chains) in any polymer follows Boltzmann distribution curve (Scheme 1) and consequently it follows the Boltzmann distribution law [16], where fraction of chains can be presented in an exponential form as follows;

$$
\begin{aligned}
& \frac{n_{i}}{N_{T}}=\frac{e^{-\frac{E_{i}}{R T}}}{\sum e^{-\frac{E_{i}}{R T}}} \\
& =e^{-\frac{\Delta E}{R T}}
\end{aligned}
$$

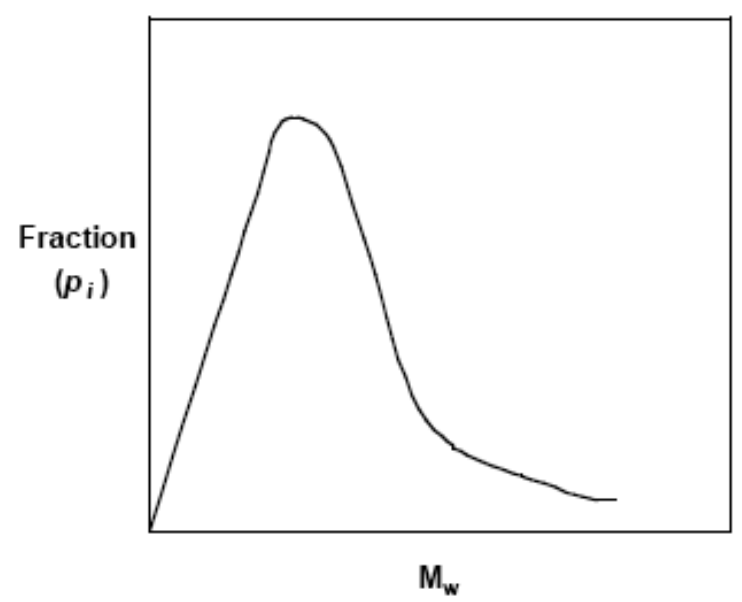

Scheme 1. Molecular weight distribution curve of polystyrene. 
For which $\boldsymbol{E}_{\boldsymbol{i}}$ presents the activation energy required for monomer to join an $\boldsymbol{i}^{\text {th }}$ length macroradical chain. The different size chains may have different activation energies (i.e. $\boldsymbol{E}_{\boldsymbol{i}}$, $\left.\boldsymbol{E}_{j}, \boldsymbol{E}_{\boldsymbol{k}}, \ldots\right)$ depends on the size length and how much entangled, coalescent, or agglomerated the chain is, in other words the activation energy differs slightly for extended chain that can coalescent, agglomerate, and largely entangled, and consequently $\Delta E$ is the resultant overall activation energy required for any monomer to join any chain of any chain length no matter what configuration does it have.

Substituting equation 8 in equation 7 turns to be:

$$
\overline{M_{n}}=\left(M_{1}+M_{2}+M_{3}+\ldots+M_{n}\right) \cdot e^{-\frac{\Delta E}{R T}}
$$

Again substituting equation 6 in equation 9 gives rise;

$$
\begin{aligned}
& \overline{M_{n}}=\left(M_{\infty} \cdot\left(1-\frac{1}{D P_{1}}\right)+M_{\infty} \cdot\left(1-\frac{1}{D P_{2}}\right)+M_{\infty} \cdot\left(1-\frac{1}{D P_{3}}\right)+\ldots+M_{\infty} \cdot\left(1-\frac{1}{D P_{n}}\right)\right) \cdot e^{-\frac{\Delta E}{R T}} \\
& =\left(\left(1-\frac{1}{D P_{1}}\right)+\left(1-\frac{1}{D P_{2}}\right)+\left(1-\frac{1}{D P_{3}}\right)+\ldots+\left(1-\frac{1}{D P_{n}}\right)\right) \cdot M_{\infty} \cdot e^{-\frac{\Delta E}{R T}}(10)
\end{aligned}
$$

Thus the molecular weight of any linear polymer at any polymerization time can accordingly be determined through the following relationship;

$$
\begin{aligned}
& M_{i, t}=\left(1-\frac{1}{D P_{i}}\right) \cdot M_{\infty} \cdot e^{-\frac{\Delta E}{R T}} \\
& \frac{M_{i, t}}{\left(1-\frac{1}{D P_{i}}\right)}=M_{\infty} \cdot e^{-\frac{\Delta E}{R T}}
\end{aligned}
$$

This linear relationship relates the momentarily change of molecular weight and degree of polymerization with the change of temperature. Hence by taking the natural logarithm for both sides;

$$
\ln \left[\frac{M_{i, t}}{\left(1-\frac{1}{D P_{i}}\right)}\right]=\ln M_{\infty}-\frac{\Delta E}{R T}
$$

This linear relationship can determine the momentarily rate of polymerization $\left(\mathbf{R}_{\mathbf{p}}\right)$ and consequently the resulting activation energy $(\Delta \mathbf{E})$ at different temperatures can be determined for this type of linear polymerization processes. Furthermore the theoretically assumed molecular weight for all monomers joining different chains at infinite time $\left(\boldsymbol{M}_{\infty}\right)$ can also be investigated and determined.

The $\boldsymbol{D} \boldsymbol{P}_{\boldsymbol{i}}$ at time zero of polymerization has a value of 1.0 (i.e. $[A]_{0=}[A]_{t}$ ), whereas at time $\infty$ it has a value of infinity $(\infty)$ (i.e. $[\mathrm{A}]_{\mathrm{t}} \cong$ zero). The determination of $\boldsymbol{D P} \boldsymbol{P}_{\boldsymbol{i}}$ can be performed through monitoring the disappearance of aliphatic $\mathrm{C}=\mathrm{C}$ for poly addition type, or monitoring the end group structural changes for poly condensation type, at different time interval through different spectroscopic techniques such as ${ }^{13} \mathrm{C}-\mathrm{NMR},{ }^{1} \mathrm{H}-\mathrm{NMR}$, FTIR, and UV-Vis, whereas the molecular weight can be determined through conventional gel permeation chromatography (GPC), Osmometry, light scattering, and Ubbelohde viscometry techniques.

Fig. (1) shows the $\ln \left[M_{i} /\left[\left(1-\left(1 / D P_{i}\right)\right]\right]\right.$ versus reciprocal temperature of polystyrene polymerized at different time interval in the temperature range of $60-80{ }^{\circ} \mathrm{C}$.

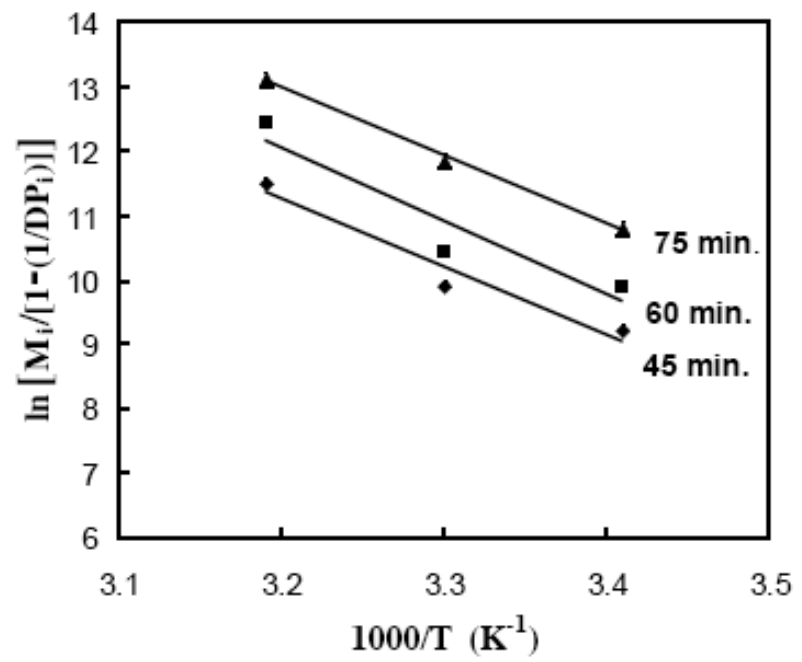

Fig. (1). $\ln \left[\mathrm{M}_{\mathrm{i}} /\left[\left(1-\left(1 / \mathrm{DP}_{\mathrm{i}}\right)\right]\right]\right.$ versus $\mathbf{1} / \mathbf{T}$ for bulk polymerized polystyrene in different time intervals in the temperature range of 60-80 ${ }^{\circ} \mathrm{C}$.

It can be clearly seen that the application of equation 12 results with linear determination of the overall activation energies at different time intervals. The overall activation energies determined from Fig. (1) range from 87-95.5 $\mathrm{kJ} / \mathrm{mol}$, which is in very good agreement with conventional determination of activation energy of different conformational polystyrenes in the literature $[17,18]$. Furthermore; the extrapolations of the curves result with almost one intercept value (i.e. $\boldsymbol{M}_{\infty}$ ) regardless to the time of polymerization, which again confirms the consistency and rigidity of equation 12 used to show and present the Arrhenius relationship in practical and renewable form.

\section{THEORETICAL APPROACH (2)}

It is known the $r_{1}=\frac{k_{11}}{k_{12}}$, where $\mathrm{k}_{11}$ and $\mathrm{k}_{12}$ are the rate constants of the interaction of a macroradical $\left(\mathrm{M}_{1}{ }^{\circ}\right)$ with either $\mathrm{M}_{1}$ or $\mathrm{M}_{2}$ monomers respectively, and $r_{2}=\frac{k_{22}}{k_{21}}$ where $\mathrm{k}_{22}$ and $\mathrm{k}_{21}$ are the rate constants of the interaction of a macroradical $\left(\mathrm{M}_{2}{ }^{\cdot}\right)$ with either $\mathrm{M}_{2}$ or $\mathrm{M}_{1}$ monomers respectively. If Arrhenius equation is applied to the reactivity ratio $r_{1}$ and $\mathrm{r}_{2}$, then the outcome will be equation 13 ;

$$
r_{1}=\frac{k_{11}}{k_{12}}=\frac{A_{11}}{A_{12}} \exp \left(\frac{E_{12}-E_{11}}{R T}\right)
$$

Consequently; $r_{2}=\frac{k_{22}}{k_{21}}$ becomes; 
Table 1. Change of $r_{1} r_{2}$ Product as Temperature Changes for (NIPAAm-alt-HEMA) Copolymers

\begin{tabular}{|c|c|c|c|}
\hline Temperature $\left.{ }^{\circ} \mathbf{C}\right)$ & $\mathbf{r}_{1}$ & $\mathbf{r}_{2}$ & $\mathbf{r}_{1} \mathbf{r}_{2}$ \\
\hline \hline 20 & 0.0034 & 0.114 & 0.0003876 \\
\hline 30 & 0.0509 & 0.427 & 0.0217343 \\
\hline 40 & 0.0356 & 0.178 & 0.0063368 \\
\hline
\end{tabular}

Table 2. Change of $\mathbf{r}_{1} \mathbf{r}_{2}$ Product as Temperature Changes for (MAAm-alt-HEMA) Copolymers

\begin{tabular}{|c|c|c|c|}
\hline Temperature $\left.{ }^{\circ} \mathbf{C}\right)$ & $\mathbf{r}_{1}$ & $\mathbf{r}_{2}$ & $\mathbf{r}_{1} \mathbf{r}_{2}$ \\
\hline \hline 5 & 0.0334 & 0.0249 & 0.0008 \\
\hline 10 & 0.0253 & 0.0252 & 0.0006 \\
\hline 20 & 0.0875 & 0.2042 & 0.0179 \\
\hline
\end{tabular}

$r_{2}=\frac{k_{22}}{k_{21}}=\frac{A_{22}}{A_{21}} \exp \left(\frac{E_{21}-E_{22}}{R T}\right)$

Thus $r_{1} r_{2}$ equals;

$r_{1} r_{2}=\frac{A_{11} A_{22}}{A_{12} A_{21}} \exp \left(\frac{\left(E_{12}-E_{11}\right)+\left(E_{21}-E_{22}\right)}{R T}\right)$

Rearrangement of equation 15 yields;

$r_{1} r_{2}=\frac{A_{11} A_{22}}{A_{12} A_{21}} \exp \left(\frac{\left(E_{12}+E_{21}\right)-\left(E_{11}+E_{22}\right)}{R T}\right)$

Equation 16 presents the linear relationship between the natural logarithm of $r_{1} r_{2}$ product $\left(\ln \left(\mathbf{r}_{1} \mathbf{r}_{2}\right)\right)$ with the reciprocal of the temperature in Kelvin (1/T), where the activation energy could be determined through the slope value of the curve. The value of the activation energy in eq' $n 16$ becomes very important because it can show the type of preference of a macroradical $\left(\mathrm{M}_{1}{ }^{\circ}\right)$ toward either $\mathrm{M}_{1}$ or $\mathrm{M}_{2}$. If $\left(\mathrm{M}_{1}{ }^{*}\right)$ macroradical prefers $M_{2}$ and $\left(M_{2}{ }^{\circ}\right)$ macroradical prefers $M_{1}$ and then the activation energy $\left(E_{12}+E_{21}\right)$ will be much smaller than $\left(E_{11}+E_{22}\right)$ and consequently the alternating behavior of copolymer will dominate. Whereas if $\left(\mathrm{M}_{1}{ }^{\circ}\right)$ macroradical prefers $\mathrm{M}_{1}$ and $\left(\mathrm{M}_{2}{ }^{\circ}\right)$ macroradical prefers $\mathrm{M}_{2}$ and then the activation energy $\left(E_{12}+E_{21}\right)$ will be much larger than $\left(E_{11}+E_{22}\right)$ and consequently a block or phase separated homopolymer behavior will dominate. Eventually if $\left(E_{12}+E_{21}\right)$ $\approx\left(E_{11}+E_{22}\right)$ then the copolymer is said to be random. Table $\mathbf{1}$ and 2 illustrate change of $r_{1} r_{2}$ product as temperature changes for both copolymers (NIPAAm-alt-HEMA) and (MAAm-alt-HEMA) [12,13] respectively. The reactivity ratios were determined using Kelen-Tudos technique [14].

Figs. (2 and 3) show the linear relationship of $\ln \left(r_{1} r_{2}\right)$ versus $1 / \mathrm{T}$, which is derived from equation 16 . The activation energy (i.e. $\left.\left(E_{12}+E_{21}\right)-\left(E_{11}+E_{22}\right)\right)$ of (NIPAAm-altHEMA) and (MAAm-alt-HEMA) copolymer were found to be $-109 \mathrm{~kJ} / \mathrm{mol}$ and $-118.4 \mathrm{~kJ} / \mathrm{mol}$ respectively. Such negative activation energies confirm that $\left(E_{12}+E_{21}\right)<<\left(E_{11}+E_{22}\right)$ in both copolymers, and hence confirms the alternating behavior of the monomers toward each other. Furthermore by comparing the activation energy values deduced from equation 16 with similar structures mentioned in the literature, it was found that they are in accordance with literature values (i.e. $\Delta \mathrm{E}=97 \pm 5 \mathrm{~kJ} / \mathrm{mol}[19]$ ).

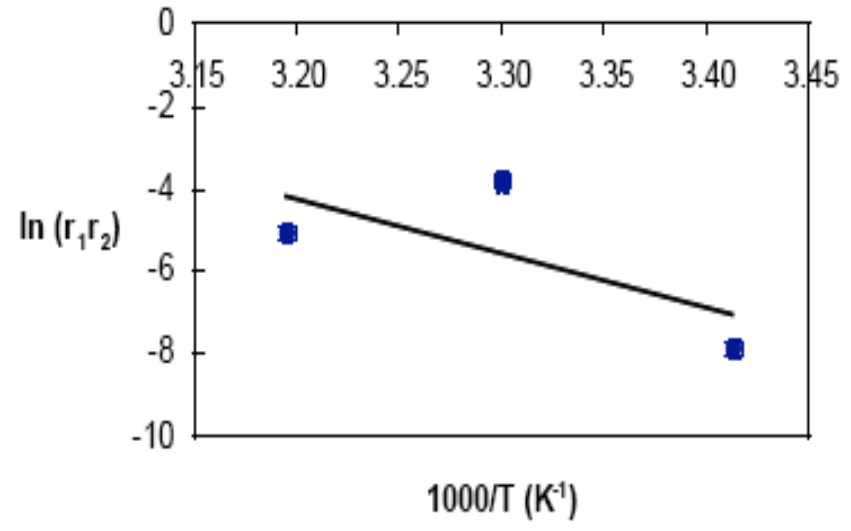

Fig. (2). Change of $\ln \left(r_{1} r_{2}\right)$ versus $1 / T$ for (NIPAAm-alt-HEMA) copolymer.

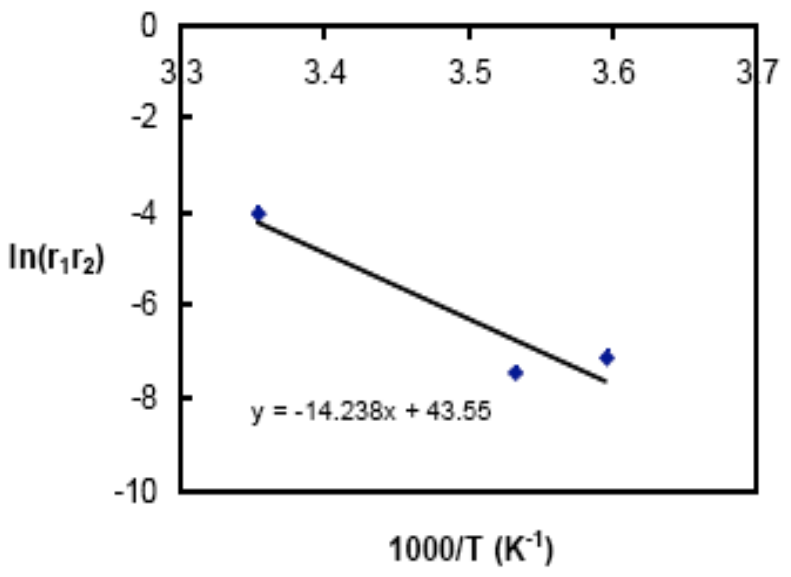

Fig. (3). Change of $\ln \left(r_{1} r_{2}\right)$ versus $1 / T$ for (MAAm-alt-HEMA) copolymer. 
From Figs. ( 2 and $\mathbf{3}$ ) it can be clearly seen that not only linear determination of activation energies can be determined but also the type of copolymers formed can be deduced and determined.

\section{CONCLUSIONS}

The two revisited mathematical kinetic relationships were derived, evaluated and eventually applied to confirm the consistency, genuinity, and linearity of the formed relationships. The first relationship, equation 12 ,

$$
\ln \left[\frac{M_{i, t}}{\left(1-\frac{1}{D P_{i}}\right)}\right]=\ln M_{\infty}-\frac{\Delta E}{R T}
$$

which relates the change of molecular weight, degree of polymerization momentarily of linear polymers with temperature, where this linear relationship can determine the overall activation energy of the polymerization process. The validity of this linear relationship was verified using different time intervals bulkily polymerized polystyrene as Fig. (1) shows. The second relationship interrelates the reactivity ratio product $\left(\mathrm{r}_{1} \mathrm{r}_{2}\right)$ with temperatures, which again showed a linear relationship, equation 16 ,

$$
r_{1} r_{2}=\frac{A_{11} A_{22}}{A_{12} A_{21}} \exp \left(\frac{\left(E_{12}+E_{21}\right)-\left(E_{11}+E_{22}\right)}{R T}\right)
$$

This linear relationship can determine the activation energy value for the copolymerization process (i.e. $\left.\left(E_{12}+E_{21}\right)-\left(E_{11}+E_{22}\right)\right)$, and consequently the type and sequence of monomers in the copolymer can be described. The validity of this equation were successfully verified for (NI-
PAAm-alt-HEMA) and (MAAm-alt-HEMA) copolymers respectively.

\section{ACKNOWLEDGMENT}

This work is acknowledged for Jordan Univeristy of Science \& Technology.

\section{REFERENCES}

[1] Matyjaszweski, K.; Davis, T. Handbook of radical polymerization, Wiley- Interscience, 2002

[2] Dong, X.; Ding, W.; Zhang, X.; Liang, X. Dyes Pigments, 2007, 74(2), 470-476.

[3] Deshmukh, S.S.; Kovalchuk, V. I.; Borovkov, V. Yu.; d'Itri, J. L. J. Phys. Chem. B, 2000, 104(6), 1277-1284.

[4] Lapidot, A.; Reuben, J.; Samuel, D. J. Chem. Edu., 1964, 41, 570.

[5] Zema, M.; Domeneghette, C.; Tazzoli, V. Am. Mineral., 1999, 84, 1895-1901.

[6] Park, J.; Lin, M. C. J. Phys. Chem., 1997, 101, 14

[7] Fares, M.M.; El-faqeeh, A. S. J. Therm. Anal. Calorimetr., 2005, $82,161-166$.

[8] Mildren, R.P.; Piper, J. A. Opt. Lett., 2003, 28, 1936-8.

[9] Carman, R. J.; Mildren R. P.; Withford M. J.; Brown, D. J. W.; Piper, J. A. IEEE J. Quantum Electron, 2000, 36, 438-49.

[10] Vollmert, B. Polymer Chemistry, Springer-Verlag, 1973, pp. 73-94.

[11] Carraher, C. E.; Seymour, R. B. Polymer Chemistry, $6^{\text {th }}$ ed. Marcel Dekker, 2003, p. 83.

[12] Fares, M. M.; Othman, A. A. J. App. Polym. Sci., accepted, 2008.

[13] Fares, M. M.; Othman, A. A. submitted, 2008.

[14] Kelen, T.; Tudos, J. Macromol. Sci. Chem. Ed., 1975, 9, 1.

[15] Carraher, C. E.; Seymour, R. B. Polymer Chemistry, $6^{\text {th }}$ ed. Marcel Dekker, 2003, p. 71.

[16] Laidler, K. J.; Meiser, J. H.; Sanctuary, B. C. Physical Chemistry, $4^{\text {th }}$ ed., Houghton Mifflin, 2003, p. 795.

[17] Jarrell, M.S.; Gates, B.C. J. Catal., 1978, 54(1), 81-93.

[18] Lupaşcu, V.; Picken, S. J.; Wübbenhorst M. Macromolecules, 2006, 39(15), $5152-5158$.

[19] Neradovich, D.; Van steenbergen, M.J.; Van steelant, L.; Meijer, Y.J.; Van Nostrum, C. F; Hennink, W. E. Macromolecules, 2003, 36, 7491-7498. 